\title{
Efecto del Ácido Láctico en la Respuesta Celular del Sistema Inmunitario Durante la Fase Inflamatoria Temprana en el Nervio Isquiático Dañado por Compresión
}

\author{
Effect of Lactic Acid on the Cellular Response of the Immune System During \\ the Early Inflammatory Phase in the Sciatic Nerve Damaged by Compression
}

\author{
Nicolás Vidal-Seguel ${ }^{1,2,3}$ \& Enrique Montiel-Eulefi ${ }^{4}$
}

VIDAL-SEGUEL, N. \& MONTIEL-EULEFI, E. Efecto del ácido láctico en la respuesta celular del sistema inmunitario durante la fase inflamatoria temprana en el nervio isquiático dañado por compresión. Int. J. Morphol., 37(4):1527-1533, 2019.

RESUMEN: En órganos dañados, el ácido láctico (AL) modifica la respuesta inmune innata e inflamatoria, induciendo una menor expresión de citoquinas pro-inflamatorias, que provocan, la modulación del reclutamiento de células inmunes. El daño por compresión del nervio isquiático (NI) desencadena una respuesta inflamatoria y un aumento exponencial del infiltrado inflamatorio de células inmunes, produciendo la destrucción de axones y pérdida funcional del nervio. El objetivo de este estudio es evaluar el efecto agudo de la inyección de AL, sobre la proporción de células inmunes en la fase inflamatoria temprana, en el sitio de lesión del NI post compresión. Para ello, se utilizaron 15 ratas machos Sprague Dawley adultas, en tres grupos de compresión nerviosa. Un grupo control, un grupo control negativo con placebo $(100 \mu \mathrm{L}$ PBS $)$ y un grupo experimental con inyección de $100 \mu \mathrm{L}$ de $\mathrm{AL}$ [20mM]. Al tercer día los NI se analizaron histológicamente y se estableció la proporción de células inmunes en el sitio de lesión. Los resultados muestran que la inyección intraneural de AL provoca una disminución en el porcentaje de linfocitos y un aumento en el porcentaje de macrófagos. Este es el primer trabajo de inyección intraneural de AL y demuestra el efecto modulador del AL sobre las células inmunes en el sistema nervioso periférico.

PALABRAS CLAVE: Nervio isquiático; Ácido láctico; Inflamación; Células inflamatorias.

\section{INTRODUCCIÓN}

El sistema nervioso periférico puede verse expuesto a patologías de tipo traumáticas, como las lesiones por aplastamiento (Fex Svennigsen \& Dahlin, 2013), que producen una exacerbada respuesta del sistema inmune innato (Gaudet et al., 2011). Por medio de los receptores de reconocimiento de patógenos (PRRs), especialmente los de la familia tolllike (TLRs) (Kawai \& Akira, 2009; Suárez \& Buelvas, 2015), que son estimulados por moléculas asociadas a daño (DAMPS) que se liberan en el tejido aplastado (Sharma \& Naidu, 2016). Las DAMPS desencadenan la activación de múltiples vías de señalización y una variedad de factores de transcripción tales como el factor nuclear kappa células B activadas (NF-kB), que regula positivamente la expresión de genes inflamatorios y la liberación de interleuquinas como, pro-IL-1b - alfa, IL-12, IL-23 y del factor de crecimiento TNF - alfa (Li et al., 2001; Tang et al., 2012), que provocan un ambiente de señalización que activa la migración leucocitaria hacia el sitio lesionado (Errea et al., 2016).

La migración leucocitaria cambia la proporción de células del sistema inmune en el sitio de lesión del nervio periférico dañado (Gaudet et al.). El primer tipo celular en migrar son los neutrófilos, considerados fundamentales en el proceso de aclaramiento del tejido dañado y amplificación inflamatoria (Nathan, 2006; Lindborg et al., 2017; Pawlina, 2018). Los neutrófilos muertos son fagocitados por macrófagos, los cuales adoptan un fenotipo anti-inflamatorio (Filardy et al., 2010). Los macrófagos en el sitio de lesión se diferencian en macrófagos endoneurales y residentes. Los endoneurales experimentan una gran proliferación durante los primeros días post lesión y los macrófagos hematógenos son quienes migran alrededor del tercer día

\footnotetext{
${ }^{1}$ Escuela de Enfermería, Facultad de Salud, Universidad Santo Tomás, Chile.

${ }^{2}$ Programa de Magíster en Ciencias mención Morfología. Facultad de Medicina. Universidad de La Frontera, Temuco, Chile.

${ }^{3}$ Becario CONICYT-PCHA/MAGÍSTER NACIONAL/ 2017-22170070.

${ }^{4}$ Laboratorio de Biomedicina, Departamento de Ciencias Básicas/CEBIOR, Facultad de Medicina, Universidad de La Frontera, Temuco, Chile.
} 
(Mueller et al., 2003). Los macrófagos desempeñan funciones fagocíticas, secretoras de factores pro-inflamatorios y presentadora de antígenos, a través, del complejo mayor de histocompatibilidad II (Mueller et al.; Pawlina). La liberación de factores pro-inflamatorios contribuye a la migración de linfocitos T, tanto CD4 como CD8, que se relacionan con la destrucción de axones amielínicos y con el dolor neuropático (Cao \& DeLeo, 2008; Zeng et al., 2013).

La respuesta inmune producto de la lesión del sistema nervioso periférico, puede causar daño secundario, al inhibir la reparación y regeneración axonal (Yuan \& Feng, 2016). Comprender los elementos de la respuesta inmune es crucial para el desarrollo de nuevas estrategias terapéuticas, que supriman las reacciones inmunes, promuevan la regeneración y restablezcan la función normal después de una lesión nerviosa (Yuan \& Feng). Ante este escenario, surge el ácido láctico como una molécula de señalización capaz modular la respuesta inmune (Sun et al., 2017).

El ácido láctico (AL) en concentraciones fisiológicas ha demostrado ser efectivo para lograr una respuesta moduladora del sistema inmune, a través, de la disminución de la síntesis de citoquinas pro-inflamatorias por parte de células del sistema inmune (Sun et al.). El AL regula negativamente la estimulación pro-inflamatoria de LPS, lo que provoca una regulación negativa de la vía de transcripción NF-kB lo que se traduce en una menor expresión de genes pro-inflamatorios, disminución de la liberación de citoquinas pro-inflamatorias y menor reclutamiento leucocitario en órganos previamente dañados (Hoque et al., 2014; Errea, et $a l$. .). La hipótesis de este estudio plantea que: la inyección intraneural de AL modula la respuesta inmune innata, por lo que afectaría la proporción de células del sistema inmmune, en la fase inflamatoria temprana en el sitio de lesión del nervio isquiático de rata post daño por compresión. Este es el primer trabajo que describe el efecto de la inyección directa de AL en el NI post compresión, sobre la proporción de células inmunes en el sitio de lesión.

\section{MATERIAL Y MÉTODO}

Animales. Para el desarrollo del trabajo con animales se cuenta con la autorización del comité de ética científica (CEC) de la Universidad de La Frontera, Temuco, Chile $\left(\mathrm{N}^{\circ}\right.$ de folio del proyecto UFRO: 043/18). Como sujetos de experimentación se utilizaron 15 ratas adultas Sprague Dawley de sexo masculino y de peso promedio de 250-300 g, agrupadas en 3 grupos de 5 animales cada uno. Las ratas fueron aportadas por el Bioterio de la Universidad de la Frontera, Temuco, Chile.
Los animales fueron alojados en jaulas individuales de policarbonato, en ambiente controlado con ciclos diarios de $12 \mathrm{~h}$ de luz/oscuridad, temperatura ambiente de $22{ }^{\circ} \mathrm{C}$, con alimento (Champion $\left.{ }^{\circledR}\right)$ y agua potable ad libitum. Estos fueron agrupados aleatoriamente en tres grupos de compresión nerviosa: 1) grupo control (GC), 2) grupo control negativo tratado con vehículo (100 $\mu \mathrm{L}$ PBS pH 7,4) (GCPBS) y 3) grupo experimental con inyección de $100 \mu \mathrm{L}$ de $\mathrm{AL}$ [20mM] en vehículo (GEAL).

Previo al protocolo quirúrgico los animales fueron anestesiados vía intraperitoneal, con $3 \mathrm{ml}$ de la mezcla ketamina/xilazina, Posterior a ello se llevó a cabo el proceso de depilación de la cara posterolateral del muslo izquierdo.

Protocolo quirúrgico. Se inició con la asepsia de la zona de abordaje y ubicación del animal sobre un campo estéril en posición decúbito lateral. Se realizó una incisión con un bisturí $n^{\circ} 24$, sobre la cara posterolateral del muslo izquierdo paralelo a la cresta iliaca de $\sim 1 \mathrm{~cm}$, se disecciono de superficial a profundo, la piel, fascia superficial, fascia profunda y finalmente la musculatura entre los músculos glúteo mayor y bíceps femoral. La separación de los vientres musculares y fascias se llevó a cabo con una tijera de disección, mientras que la exposición del nervio isquiático se realizó con una pinza anatómica. Los animales fueron posicionados sobre una máquina construida de acero inoxidable capaz de inducir daño nervioso por medio de un peso controlado. Se aplicó sobre el nervio isquiático izquierdo una carga de 15 $\mathrm{kg}$ durante 10 minutos con un área de trituración circular $\sim 0,2826 \mathrm{~cm} 2$ (Ø 0,6 cm, $5.2 \mathrm{MPa}, 53.07 \mathrm{kgf} / \mathrm{cm}^{2}$ ) (Schiaveto de Souza et al., 2004).

Los grupos GCPBS y GEAL fueron sometidos a una inyección intraneural en el sitio de lesión de $100 \mu \mathrm{L}$ de PBS y de $100 \mu \mathrm{L}$ AL [20 mM], respectivamente. La inyección intraneural fue realizada inmediatamente posterior a la lesión siguiendo lo descrito por Kim et al. (2018). Tras la lesión el nervio isquiático se reposicionó en su lugar habitual, la incisión fue cerrada por planos y la piel cerrada con sutura de nylon 4-0 y tratada con antiséptico en espray. Durante los tres días post operatorio los animales fueron tratados con Buprenorfina vía subcutánea, en dosis de $0,1 \mathrm{mg} / \mathrm{kg}$, cada 12 horas.

Recolección y procesamiento de las muestras. Al tercer día post operatorio, los animales se sacrificaron en una cámara de Dióxido de carbono $\left(\mathrm{CO}_{2}\right)$ con regulador prefijado a 5 psi, seguido de la disección y recolección del tejido. Las muestras de $4 \mathrm{~mm}$ de nervio isquiático fueron inmersas en

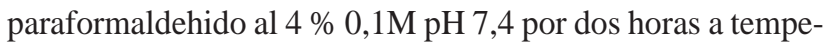
ratura ambiente y luego a $4{ }^{\circ} \mathrm{C}$ por tres días. La deshidrata- 
ción se realizó en una batería creciente de alcohol etílico desde $50 \%$ a $100 \%$. La inclusión fue realizada con Paraplast plus ${ }^{\circledR}$ y los tacos fueron tallados con los nervios orientados para obtener cortes longitudinales. De cada taco se realizaron cortes seriados de 5 micras, los que fueron montados en portaobjetos tratados previamente poli-L-lisina $(0,1 \%)$ y teñidos con azul de toluidina. Se optó por este colorante debido a su baja afinidad por el tejido fibroso lo que facilita la identificación morfológica de células inmunes en el sito de lesión.

Análisis morfológico. El análisis morfológico se realizó mediante la observación en un microscopio Leica DM750 con cámara Leica ICC50 HD, facilitado por el Doctorado en Morfología de la Universidad de La Frontera. El análisis cualitativo de las muestras se realizó mediante la observación microscópica a diferentes aumentos y se esquematizó en 3D por el programa Blender 3D. El análisis cuantitativo de la proporción de células inmunes en el sitio de lesión se realizó de la siguiente manera: 1) El sitio de lesión se consideró como el espacio comprendido entre los segmentos proximal y distal, caracterizado por presentar una baja coloración y gran presencia de células inflamatorias y 2) la cuantificación de las células inmunes se realizó a través de un barrido del sitio de la lesión, donde se estableció la proporción en porcentaje de células del sistema inmunitario: macrófagos, linfocitos, neutrófilos y mastocitos de 100 células del sitio de lesión (Mueller et al.).

Análisis estadístico. El análisis estadístico se realizó utilizando el paquete estadístico para las ciencias sociales SPSS (IBM SPSS Statistics, v 21). Para conocer la normalidad de los datos se utilizó la prueba de Shapiro-Wilk. Los datos con distribución normal, se analizaron mediante ANOVA de una vía $(\mathrm{p}<0,05)$ y la prueba post hoc de TUKEY HSD. Para los datos sin distribución normal, se utilizó el test no paramétrico de Kruskal-Wallis.

\section{RESULTADOS}

Descripción histológica. La lesión se presenta externamente como una zona abultada y enrojecida, con dos zonas extremas denominadas segmento distal y proximal. La histología de los nervios lesionados teñidos con azul de toluidina permite identificar tres zonas: dos extremos que corresponden a los segmentos distal y proximal y una zona central que corresponde al sito de lesión (Figs. 1A y 1B).

El área de sitio de lesión carece de un patrón ondulatorio, por la falta de fibras nerviosas debido a la compresión, este espacio comienza a ser ocupado por tejido fibroso y distintos tipos celulares distribuidos en toda la lesión. Este sitio adopta un color celeste tenue a la tinción con azul de toluidina, ya que este colorante posee una baja afinidad con el tejido conectivo y colágeno, favoreciendo así la identificación morfológica de macrófagos, neutrófilos, linfocitos y mastocitos.

Los macrófagos se observan como células de núcleo circular o arriñonado y de un citoplasma tortuoso, con una gran presencia al tercer día post compresión (Fig. 1C). Los linfocitos se observan como células redondeadas de coloración intensa y escaso citoplasma (Fig. 1D), con una gran presencia en el sitio de lesión y en el espacio extraneural. Los neutrófilos se observan como células de núcleo lobulado y escasa tinción citoplasmática (Fig. 1E), su presencia en el sitio de lesión al tercer día post compresión es abúndate, sin embargo, disminuyen hacia segmentos más distales. Los mastocitos se observan como células con gránulos de color violeta, producto de la metacromasia a la tinción con azul de toluidina, sin embargo, su presencia en el sitio de lesión es escasa (Fig. 1F).

Descripción cuantitativa. Los promedios de las proporciones de cada tipo celular fueron obtenidos de 5 ratas de cada grupo, su comparación con el test de ANOVA muestra la existencia de diferencias significativas, $p<0,005$ en macrófagos y linfocitos (Tabla I), planteando que el AL produce una variación en la respuesta inmune y en la proporción celular. La Figura 2 muestra la proporción de células inmunes en el sitio de la lesión del NI al tercer día post compresión, donde, la proporción de macrófagos aumenta significativamente en el grupo tratado con AL, 38,0 \%, respecto de los grupos control con lesión sin inyectar, 26,2 \% y el tratado con PBS como vehículo, 23,6 \%. La proporción de linfocitos baja en el grupo tratado con AL, 22,0 \%, comparado con el tratado con vehículo, $31,8 \%$ y sin inyectar $28,2 \%$. Por otra parte, los neutrófilos mantienen una proporción similar entre los grupos, de 41,8 y 45, $6 \%$, p=0,64. Los mastocitos son escasos y no muestran diferencias entre los distintos grupos de experimentación: GC: $0.04 \%$; GCPBS: $0.04 \%$ y GEAL: $0.08 \%$ con Kruskal-Wallis no significativo.

\section{DISCUSIÓN}

Debido a la complejidad y alta prevalecía de los trastornos neurológicos, sumado al actual rol del AL en la inflamación, se plantea la hipótesis de que el AL podría afectar la proporción celular en la fase inflamatoria temprana en el sitio de lesión del nervio isquiático de rata post compresión. Esto con el objetivo futuro de buscar nuevas alternativas que mejoren la reparación nerviosa posterior a una lesión. 

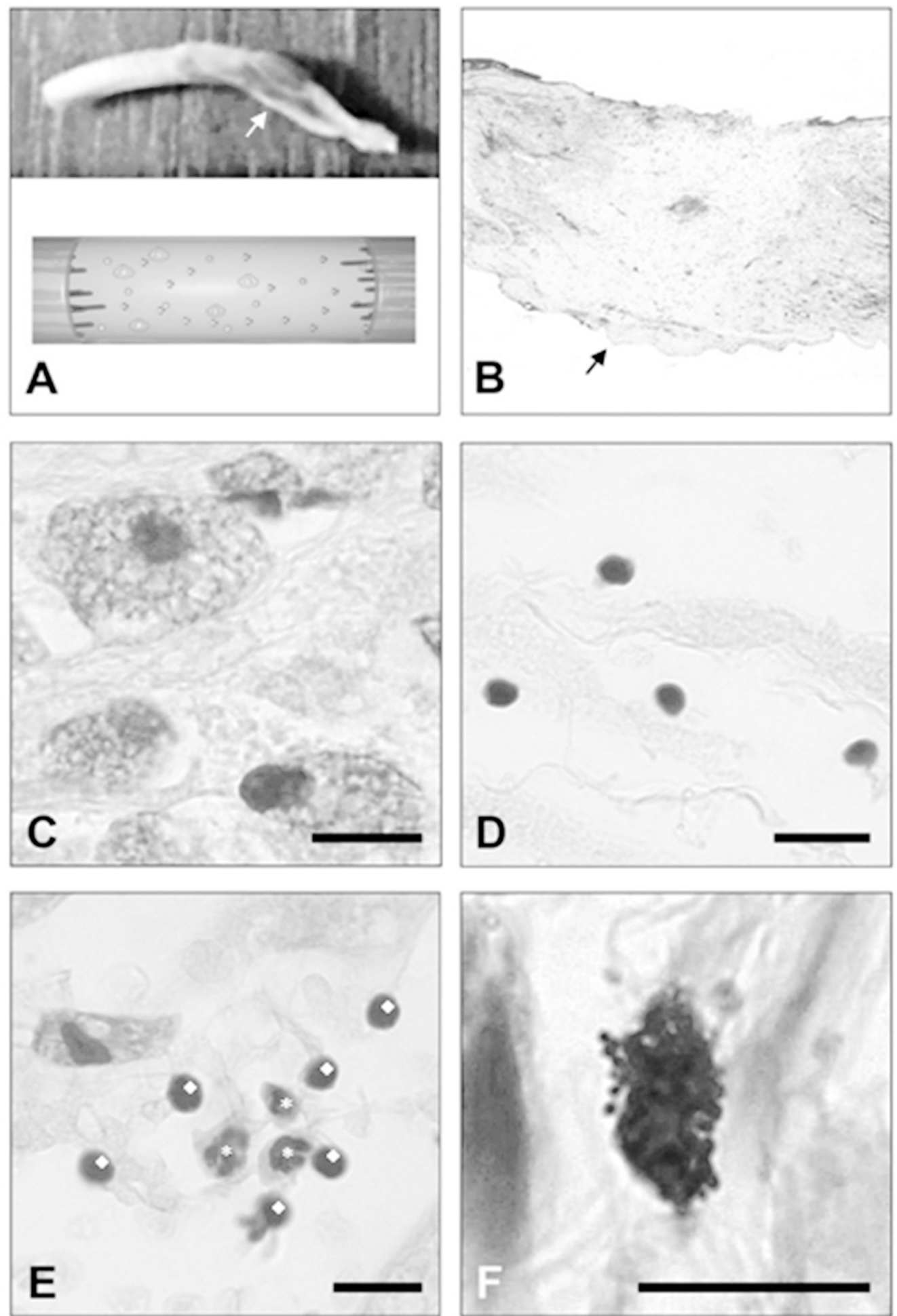

Fig. 1. Análisis de la lesión NI y tipos celulares observados al tercer día post compresión. A) Imagen superior muestra la sección del NI que presenta un hematoma interneural (flecha) y en inferior se muestra un esquema del sitio de lesión que presenta la distribución relativa de los tipos celulares. B) Microfotografía del corte histológico del NI post compresión, nótese la poca tinción del sitio de la lesión (flecha). C) Microfotografía que muestra macrófagos, D) Microfotografía que muestra linfocitos. E) Microfotografía que muestra neutrófilos $(*)$ y linfocitos $(\diamond)$. F) Microfotografía que muestra los gránulos típicos de un mastocito. Barra $=10 \mu \mathrm{m}$. 


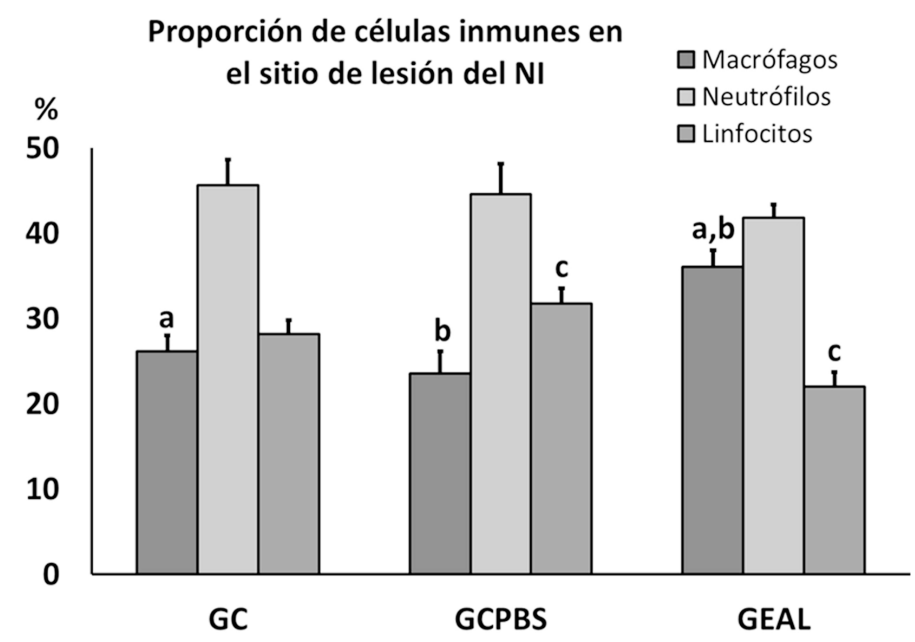

Fig. 2. El gráfico muestra los cambios en la proporción de células inmunes en el sitio de lesión, causadas por la inyección de AL en la fase inflamatoria temprana. La cuantificación de los distintos tipos celulares durante la fase inflamatoria temprana 3 días después del tratamiento en 5 ratas diferentes por grupo. Los controles sin tratamiento (GC) y con inyección de vehículo (GCPBS) son comparados con la inyección experimental de AL (GEAL). Los resultados muestran un aumento estadísticamente diferente en la proporción de macrófagos en los nervios tratados con AL (GEAL ?:38,04\%), respecto a cada control: GC (?:26,16 $\%$; a: $\mathrm{p}=0,02)$ y GCPBS $(?: 23,56 \% ; \mathrm{b}: \mathrm{p}=0,004)$. La proporción de linfocitos en los nervios tratados con AL (GEAL ?:22,0\%) muestran una reducción estadísticamente significativa respecto al control GCPBS (?:31,8 $\%$; $\mathrm{c}$ : $\mathrm{p}=0,004)$ y con una similar tendencia a la disminución con el control: GC (?:28,2\% p=0.06) sin llegar a la diferencia estadísticamente significativa. Los neutrófilos muestran una similar proporción entre los distintos grupos GEAL (?:41,84\%), GC (?:45,64\%) y GCPBS (?:44,56\%) sin encontrar diferencia estadísticamente significativa (ANOVA $\mathrm{p}=0,64$ ). realiza y no existe otro estudio similar con el cual llevar a cabo una comparación, sin embargo, la concentración escogida se asemeja a la alcanzada fisiológicamente durante la práctica de ejercicio intenso de corta duración (Parsikia et al., 2014) y se encuentra dentro del rango considerado efectivo para lograr un efecto sobre la modulación de la expresión de citoquinas proinflamatorias por células del sistema inmune en hígado y páncreas (Hoque et al.)

La lesión nerviosa por compresión ocasiona en el nervio lesionado un hematoma intraneural (Scopel et al., 2007) que microscópicamente se caracteriza por una serie de cambios e interacciones celulares que rigen los procesos de degeneración Walleriana y posterior reparación nerviosa (Pawlina; Wu et al.). estos procesos que se inician en el sitio de lesión inicio se caracterizan por la proliferación de macrófagos residentes y neurolemocitos, así como, el aclaramiento de la zona lesionada y la migración leucocitaria (Gaudet et al.; Pawlina). Esta descripción concuerda con lo observado, donde, el sitio de lesión presenta un color celeste pálido a la tinción con azul de toluidina, debido a la ausencia de fibras nerviosas y a la baja afinidad que este posee con el tejido fibroso, además, se destacan una cantidad importante de células inmunes distribuidas en el tejido lesionado (Yuan \& Feng).

El nervio indemne presenta un bajo porcentaje de células inmunes, sin embargo, posterior a una lesión, estas células experimentan un incremento exponencial, como producto de la proliferación y migración hacia el sitio de lesión (Mueller et al.). troles (GC y GCPBS).

\begin{tabular}{lccl}
\hline Tipo celular & Normalidad SW $\mathrm{n}<30$ & ANOVA & Tukey HSD \\
\hline Macrófagos & $\mathrm{p}>0,05$ & $\mathrm{p}=0,004$ & GC y GEAL $(\mathrm{p}=0,02)$ \\
& $\mathrm{p}>0,05$ & $\mathrm{p}=0,64$ & - \\
Neutrófilos & $\mathrm{p}>0,05$ & $\mathrm{p}=0,005$ & GCPBS y GEAL $(\mathrm{p}=0,004)$ \\
Linfocitos & $\mathrm{p}<0,05$ & - & - \\
Mastocitos & & & \\
\hline
\end{tabular}

En nuestro estudio se inyecto AL intraneural al nervio isquiático posterior a la compresión, siguiendo el protocolo de Kim et al., en el cual se inyecto $200 \mu \mathrm{L}$ de dexmedetomidina post lesión. En el presente estudio un volumen $100 \mu \mathrm{L}$ fue suficiente para ocasionar un aumento considerable del volumen intraneural en el sitio de lesión evitando generar un daño adicional. Este volumen se asemeja al elegido por Wu et al (2018) en donde se expuso al nervio a inyecciones intraneurales de $6 \mu \mathrm{l}$ a $150 \mu \mathrm{L}$ de ATP.

La concentración de $20 \mathrm{mM}$ de AL inyectada al NI, como dosis única en este estudio es la primera vez que se
Los macrófagos observados en este estudio se distribuyen ampliamente en el sitio de lesión y en los segmentos adyacentes, Stoll et al. (1989) atribuye este desplazamiento a las señales locales que dirigen a los macrófagos a distintas áreas del nervio lesionado.

Los linfocitos observados se distribuyen en el sitio de lesión, en el espacio extraneural y en el segmento distal Moalem et al. (2004), describen que los linfocitos comienzan a migrar al tercer día y alcanzan su punto más alto entre los días 14 y 28 post lesión La gran proporción de linfocitos encontrados en este trabajo sugiere que el inicio de esta migración es anterior a la fecha indicada debido a la gran proporción de linfocitos encontrados al tercer día post lesión, sin embrago, coincidimos en que el punto más alto se logra con posterioridad, debido al gran número de linfocito ubi- 
cados extraneuralmente que aún no han migrado al sitio lesionado (Datos no mostrados).

Nuestra observación de los neutrófilos coincide a lo descrito por Nathan, quien describe a este tipo celular como el más abundante de la primera ola de migración hacia el órgano afectado y a lo expuesto por Moalem et al., quienes describen un aumento sustancial de neutrófilos desde las 8 horas con un aumento importante dentro de las primeras 24 horas post lesión, además de una distribución escasa hacia áreas más distales.

La observación de mastocitos los posiciona como el tipo celular menos abundante en el sitio de lesión a los tres días post compresión. Los resultados obtenidos coinciden a los expuesto por Enerbaeck et al. (1965), quienes reportaron que los mastocitos están completamente ausentes en el sitio de lesión durante las primeras semanas, sin embargo, existe un aumento exponencial dentro de la cuarta a octava semana post lesión.

El efecto del AL sobre la respuesta inmune en tejidos fue expuesta cualitativamente por Hoque et al. quienes evidenciaron una disminución de macrófagos y neutrófilos en hígado y páncreas de ratas intervenidas, como producto de la inyección intraperitoneal de AL, sin embargo, esta descripción histológica es más bien una apreciación de un tejido sin mayores detalles estadísticos. En nuestro estudio los datos cuantitativos muestran que no existen diferencias en la proporción de neutrófilos entre grupos, pero si un aumento de macrófagos en el GEAL respecto a los grupos controles, este dato se contradice con lo expuesto por Hoque et al. Lo que puede ser explicado, pues el aumento en la proporción de macrófagos en el sitio de lesión no es comparable al número absoluto, así como, el aumento de la proporción de macrófagos puede ser consecuencia de la disminución de la proporción de linfocitos o considerando que el sistema nervioso periférico se comporta distinto a los otros órganos frente a la agresión y donde el AL pudo haber retrasado la migración de macrófagos hacia el sitio de lesión y con ello su desplazamiento hacia áreas más distales, lo que se traduce en una mayor proporción en el sitio de lesión a la hora de la evaluación. Otra posible explicación es que los macrófagos residentes pudieron haber potenciado su proliferación, ideas que son motivo de estudio, actualmente se sabe que los macrófagos residentes poseen un origen embriológico distinto a los hematógenos (Varol et al., 2015), pudiendo ser influenciados por el AL como lo sugiere datos no publicados de nuestro laboratorio. La caracterización de la proporción de macrófagos residentes, hematógenos o con fenotipo M1 o M2 en el sitio de lesión es motivo de futuras investigaciones.
Nuestros datos también muestran una menor proporción de linfocitos en el GEAL con respecto a los grupos controles. Los linfocitos han sido relacionados con la posibilidad de destrucción de axones amielínicos mediante la producción de citocinas neurotóxicas y con el dolor neuropático (Zeng et al.). La menor proporción de linfocitos obtenida en este estudio puede indicar una menor destrucción axonal y un mejor escenario para la reparación del NI, sin embargo, son necesarias mayores investigaciones para comprobar este beneficio, así como, caracterizar el fenotipo de los linfocitos dentro del sitio de lesión.

Los resultados obtenidos en este estudio son un primer acercamiento al efecto que posee el AL en la fase inflamatoria temprana en el NI post lesión. La existencia de una diferencia en la proporción de células inmunes con un aumento de macrófagos y una disminución de linfocitos en el sitio de lesión al tercer día post compresión son datos relevantes, teniendo en cuenta que cada tipo celular cumple un rol especifico en la reparación nerviosa, sin embargo, son necesarios más estudios sobre el papel de cada tipo celular en la reparación nerviosa y el rol que puede jugar el AL en este complejo proceso.

\section{AGRADECIMIENTOS}

Este trabajo fue financiado por la Universidad de La Frontera, proyecto DI14-0051, Temuco, Chile. N. Vidal fue Becario de Magíster, financiado por CONICYT, PFCHA/ MAGISTER NACIONAL/2017 - 22170070, Chile. Al Dr. Fernando Días y al Mg. Emilio Farfán, por su ayuda en el diseño experimental y al Doctorado en Ciencias Morfológicas y su director Dr. Mariano De Sol por facilitar el uso de sus espacios y del equipamiento del programa.

VIDAL-SEGUEL, N. \& MONTIEL-EULEFI, E. Effect of lactic acid on the cellular response of the immune system during the early inflammatory phase in the sciatic nerve damaged by compression. Int. J. Morphol., 37(4):1527-1533, 2019.

SUMMARY: In damaged organs, lactic acid (LA) modifies the innate and inflammatory immune response, inducing a lower expression of pro-inflammatory cytokines, which provoke the modulation of immune cell recruitment. Damage by compression of the sciatic nerve $(\mathrm{SN})$ triggers an inflammatory response and an exponential increase in the inflammatory infiltrate of immune cells, producing the destruction of axons and functional loss of the nerve. The objective of this study is to evaluate the acute effect of the injection of LA, on the proportion of immune cells in the early inflammatory phase, in the site of SN post-compression injury. For this, 15 adult Sprague Dawley rats were used in three groups of nervous compression. A control group, a negative control group 
with placebo (100 mL PBS) and an experimental group with injection of $100 \mathrm{~mL}$ of LA [20mM]. On the third day, the SNs were histologically analyzed and the proportion of immune cells at the injury site was established. The results show that the intraneural injection of LA causes a decrease in the percentage of lymphocytes and an increase in the percentage of macrophages. This is the first work of intraneural injection of LA and demonstrates the modulating effect of LA on immune cells in the peripheral nervous system.

KEY WORDS: Sciatic nerve; Lactic acid; Inflammation; Inflammatory cells.

\section{REFERENCIAS BIBLIOGRÁFICAS}

Cao, L. \& DeLeo, J. A. CNS-infiltrating CD4+ T lymphocytes contribute to murine spinal nerve transection-induced neuropathic pain. Eur. J. Immunol., 38(2):448-58, 2008.

Enerbaeck, L.; Olsson, Y. \& Sourander, P. Mast cells in normal and sectioned peripheral nerve. Z. Zellforsch. Mikrosk. Anat., 66(4):596-608, 1965.

Errea, A.; Cayet, D.; Marchetti, P.; Tang, C.; Kluza, J.; Offermanns, S.; Sirard, J. C. \& Rumbo, M. Lactate inhibits the pro-inflammatory response and metabolic reprogramming in murine macrophages in a GPR81-independent manner. PLoS One, 11(11):e0163694, 2016.

Fex Svennigsen, A. \& Dahlin, L. B. Repair of the peripheral nerveremyelination that works. Brain Sci., 3(3):1182-97, 2013.

Filardy, A. A.; Pires, D. R.; Nunes, M. P.; Takiya, C. M.; Freire-de-Lima, C. G.; Ribeiro-Gomes, F. L. \& DosReis, G. A. Proinflammatory clearance of apoptotic neutrophils induces an IL-12(low)IL-10(high) regulatory phenotype in macrophages. J. Immunol., 185(4):2044-50, 2010.

Gaudet, A. D.; Popovich, P. G. \& Ramer, M. S. Wallerian degeneration: gaining perspective on inflammatory events after peripheral nerve injury. J. Neuroinflammation, 8:110, 2011.

Hoque, R.; Farooq, A.; Ghani, A.; Gorelick, F. \& Mehal, W. Z. Lactate reduces liver and pancreatic injury in Toll-like receptor- and inflammasome-mediated inflammation via GPR81-mediated suppression of innate immunity. Gastroenterology, 146(7):1763-74, 2014.

Kawai, T. \& Akira, S. The roles of TLRs, RLRs and NLRs in pathogen recognition. Int. Immunol., 21(4):317-37, 2009.

Kim, B. S.; Choi, J. H.; Baek, S. H. \& Lee, D. H. Effects of intraneural injection of dexmedetomidine in combination with ropivacaine in rat sciatic nerve block. Reg. Anesth. Pain Med., 43(4):378-84, 2018.

Li, M.; Carpio, D. F.; Zheng, Y.; Bruzzo, P.; Singh, V.; Ouaaz, F.; Medzhitov, R. M. \& Beg, A. A. An essential role of the NF-kappa B/Toll-like receptor pathway in induction of inflammatory and tissue-repair gene expression by necrotic cells. J. Immunol., 166(12):7128-35, 2001.

Lindborg, J. A.; Mack, M. \& Zigmond, R. E. Neutrophils are critical for myelin removal in a peripheral nerve injury model of wallerian degeneration. J. Neurosci., 37(43):10258-77, 2017.

Moalem, G.; Xu, K. \& Yu, L. T lymphocytes play a role in neuropathic pain following peripheral nerve injury in rats. Neuroscience, 129(3):76777, 2004.

Mueller, M.; Leonhard, C.; Wacker, K.; Ringelstein, E. B.; Okabe, M.; Hickey, W. F. \& Kiefer, R. Macrophage response to peripheral nerve injury: the quantitative contribution of resident and hematogenous macrophages. Lab. Invest., 83(2):175-85, 2003.

Nathan, C. Neutrophils and immunity: challenges and opportunities. Nat. Rev. Immunol., 6(3):173-82, 2006.

Parsikia, A.; Bones, K.; Kaplan, M.; Strain, J.; Leung, P. S.; Ortiz, J. \&
Joshi, A. R. The predictive value of initial serum lactate in trauma patients. Shock, 42(3):199-204, 2014.

Pawlina, W. Histology. A Text and Atlas with Correlated Cell and Molecular Biology. $7^{\text {th }}$ ed. Philadelphia, Lippincott Williams \& Wilkins, 2018.

Schiaveto de Souza, A.; da Silva, C. A. \& Del Bel, E. A. Methodological evaluation to analyze functional recovery after sciatic nerve injury. $J$. Neurotrauma, 21(5):627-35, 2004.

Scopel, G. P.; Faria, J. C. M.; Busnardo, F. F.; Alves, H. R. N.; Orpheu, S. C. \& Ferreira, M. C. Experimental intraneural hematoma model in rats: evaluation of functional recovery and neural histomorphometry. Acta Ortop. Bras., 15(4):222-6, 2007.

Sharma, S. K. \& Naidu, G. The role of danger-associated molecular patterns (DAMPs) in trauma and infections. J. Thorac. Dis., 8(7):1406-9, 2016.

Stoll, G.; Griffin, J. W.; Li, C. Y. \& Trapp, B. D. Wallerian degeneration in the peripheral nervous system: participation of both Schwann cells and macrophages in myelin degradation. J. Neurocytol., 18(5):671-83, 1989.

Suárez, R. \& Buelvas, N. El inflamasoma: mecanismos de activación. Investig. Clin., 56(1):74-99, 2015.

Sun, S.; Li, H.; Chen, J. \& Qian, Q. Lactic acid: No longer an inert and end-product of glycolysis. Physiology (Bethesda), 32(6):453-63, 2017.

Tang, D.; Kang, R.; Coyne, C. B.; Zeh, H. J. \& Lotze, M. T. PAMPs and DAMPs: signal 0 s that spur autophagy and immunity. Immunol. Rev., 249(1):158-75, 2012.

Varol, C.; Mildner, A. \& Jung, S. Macrophages: development and tissue specialization. Anпu. Rev. Immunol., 33:643-75, 2015.

Wu, D.; Lee, S.; Luo, J.; Xia, H.; Gushchina, S.; Richardson, P. M.; Yeh, J.; Krügel, U.; Franke, H.; Zhang, Y.; et al. Intraneural injection of ATP stimulates regeneration of primary sensory axons in the spinal cord. $J$. Neurosci., 38(6):1351-5, 2018.

Yuan, W. \& Feng, X. Immune cell distribution and immunoglobulin levels change following sciatic nerve injury in a rat model. Iran. J. Basic Med. Sci., 19(7):794-9, 2016.

Zeng, X. L.; Nagavalli, A.; Smith, C. J.; Howard, J. F. \& Su, M. A. Divergent effects of T cell costimulation and inflammatory cytokine production on autoimmune peripheral neuropathy provoked by Aire deficiency. $J$. Immunol., 190(8):3895-904, 2013.

\author{
Dirección para correspondencia: \\ Montiel-Eulefi, E. \\ Universidad de La Frontera \\ Facultad de Medicina \\ Centro de Biotecnología en Reproducción, CEBIOR \\ Montevideo 0870 \\ Temuco 4811322 \\ CHILE
}

Email: enrique.montiel@ufrontera.cl

Recibido : 15-03-2019

Aceptado: 06-06-2019 